\title{
Temperature dependence of atomic vibrations in mono-layer graphene
}

\author{
Christopher S. Allen, ${ }^{1, a)}$ Emanuela Liberti, ${ }^{1}$ Judy S. Kim, ${ }^{1}$ Qiang $\mathrm{Xu},{ }^{2}$ Ye Fan, ${ }^{1}$ Kuang He, ${ }^{1}$ \\ Alex W. Robertson, ${ }^{1}$ Henny W. Zandbergen, ${ }^{3}$ Jamie H. Warner, ${ }^{1}$ and Angus I. Kirkland ${ }^{1}$ \\ ${ }^{1}$ Department of Materials, University of Oxford, Parks Road, Oxford OX1 3PH, United Kingdom \\ ${ }^{2}$ DENs Solutions, Delftechpark 26, 2628 XH Delft, The Netherlands \\ ${ }^{3}$ Kavli Institute of Nanoscience, Delft University of Technology, 2628 CJ Delft, The Netherlands
}

(Received 21 May 2015; accepted 29 July 2015; published online 19 August 2015)

\begin{abstract}
We have measured the mean square amplitude of both in- and out-of-plane lattice vibrations for mono-layer graphene at temperatures ranging from $\sim 100 \mathrm{~K}$ to $1300 \mathrm{~K}$. The amplitude of lattice vibrations was calculated from data extracted from selected area electron diffraction patterns recorded across a known temperature range with over 80 diffraction peaks measured per diffraction pattern. Using an analytical Debye model, we have also determined values for the maximum phonon wavelength that can be supported by a mono-layer graphene crystal and the magnitude of quantum mechanical zero point vibrations. For in-plane phonons, the quantum mechanical zero point contribution dominates the measured atomic displacement at room temperature, whereas for out-of-plane modes, thermally populated phonons must be considered. We find a value for the maximum phonon wavelength sampled that is several orders of magnitudes smaller than the physical crystallite size. C 2015 AIP Publishing LLC. [http://dx.doi.org/10.1063/1.4928324]
\end{abstract}

\section{INTRODUCTION}

In low dimensional materials, it has been theoretically argued that long range crystalline order should break down in the presence of arbitrarily small atomic vibrations. ${ }^{1-3}$ With the isolation of graphene ${ }^{4}$ and other low-dimensional materials, ${ }^{5-7}$ this argument has been experimentally refuted provoking questions regarding the nature of phonons and crystalline order in this class of material. Atomistic Monte Carlo simulations have suggested that a two-dimensional atomic sheet can be stabilized if rippling normal to the sheet plane is considered ${ }^{8,9}$ and such long-range rippling has been experimentally observed in mono-layer graphene. ${ }^{10}$

In this work, we have studied a measure of long range crystalline order in mono-layer graphene: the Debye-Waller factor (DWF). Using electron diffraction, we have measured the DWF in mono-layer graphene at temperatures approaching the Debye temperature for flexural (normal to basal plane) phonon modes.

For static atoms in a perfect lattice, the intensity of a Bragg reflection or diffraction spot is determined solely by the atomic form factor of the constituent atom and the crystalline periodicities within the lattice. ${ }^{11}$ However, atoms are not static (even at zero Kelvin) and any motion causes a reduction in the intensity of the Bragg diffracted peaks in a mechanism by which intensity is redistributed into a diffuse background. This intensity reduction is known as the DebyeWaller factor and can be mathematically described as an exponential decay $\exp (-2 W),{ }^{12}$ where $2 W$ is the quantum statistical-mechanical average of the product of scattering vector and mean square atomic displacement.

Recent theoretical work ${ }^{13}$ has shown that for the case of two-dimensional crystals, the DWF diverges at any finite

\footnotetext{
a) Author to whom correspondence should be addressed. Electronic mail: christopher.allen@materials.ox.ac.uk
}

temperature corresponding to the predicted break-down of crystalline order in low dimensional materials. ${ }^{1-3}$ However, at temperatures much lower than the Debye temperature (the temperature of the crystals highest normal mode of vibration), this singularity in the DWF is avoided if a limit is placed on the maximum phonon wavelength that the twodimensional crystal can support. ${ }^{13,14}$

Knowledge of the DWF in low dimensional materials, such as mono-layer graphene, not only provides information about the nature of long range crystalline order but also aids our understanding of scattering processes in these materials which is essential to any quantitative description of image formation in transmission electron microscopy. ${ }^{15,16}$

\section{METHODS}

Mono-layer graphene was grown by chemical vapour deposition (CVD) and transferred to TEM grids using a polymer scaffold ${ }^{17}$ as described previously. To perform heating experiments, mono-layer graphene was transferred to commercially available chips (DENS-C-SH30), incorporating a platinum heating coil ${ }^{18}$ in which slits of $0.2 \times 1 \mu \mathrm{m}$ had been drilled using a focused ion beam. Selected area diffraction patterns were recorded using a monochromated dual aberration corrected JEOL-2200 MCO TEM, ${ }^{19}$ operating at $80 \mathrm{kV}$ with an electron beam energy spread of $\sim 380 \mathrm{meV}$. To perform in-situ heating experiments, a SH30-4M-FS (DENS solutions) TEM sample holder was used. Cooling experiments were performed using a liquid nitrogen TEM sample holder manufactured by Fischione Instruments. ${ }^{20}$ Selected area diffraction patterns were recorded on a $4096 \times 4096$ pixel Gatan Ultrascan CCD using an aperture of $\sim 360 \mathrm{~nm}$ diameter in the image plane, a $20 \mathrm{~cm}$ camera length, and an exposure time of $4 \mathrm{~s}$. 


\section{RESULTS AND DISCUSSION}

Figure 1(a) shows a selected-area electron diffraction (SAED) pattern from suspended mono-layer graphene. The ratio of the integrated intensities of the $(0, \overline{1}, 1,0)$ and $(\overline{1}, 0,1,0)$ to the $(1, \overline{2}, 1,0)$ and $(\overline{2}, 1,1,0)$ reflections is $\sim 1.1$, confirming that the graphene sample is predominantly mono-layer. ${ }^{21}$ Reflections were recorded to scattering angles greater than $G=6 G_{0}$, where $G_{0}$ is the modulus of the reciprocal lattice vector, $b_{1}$. The weakest reflections recorded had a signal to noise ratio $\sim 6$, exceeding the Rose criterion for the detection of features in a noisy signal. ${ }^{22}$ Figure 1(b) shows a typical TEM image of the mono-layer graphene sample recorded at room temperature, illustrating the overall sample cleanliness with contamination free areas of the order of a few hundred $\mathrm{nm}^{2}$.

Using kinematical scattering theory, Shevitski and coworkers have derived ${ }^{14}$ an analytical expression for the number of electrons scattered into a reflection $\left(N_{\text {peak }}\right)$ at a scattering vector $\Delta \boldsymbol{k}$ as

$$
N_{\text {peak }}=\frac{16}{27} \frac{I \tau}{e}\left(\frac{Z \gamma \lambda}{a_{B}}\right)^{2}\left|S_{b}\left(v_{1}, v_{2}\right)\right|^{2} \frac{e^{-2 W}}{\delta^{4}\left(\mu^{2}+\Delta \boldsymbol{k}^{2}\right)^{2}} .
$$

In (1), the incident electrons are described in terms of the total electron dose (the product of beam current $I$ and exposure time $\tau$ ) and constants related to the characteristics of the electron beam $(\gamma$, the Lorentz factor and $\lambda$ the wave length of incident radiation). Scattering from the mono-layer graphene lattice is taken into account through the lattice sum $S_{b}\left(v_{1}, v_{2}\right)$ and specific material parameters ( $Z$ the atomic number of carbon, $a_{B}$ the electron Bohr radius, $\delta$ the carboncarbon bond length in mono-layer graphene, $\mu$ an inverse Coulomb screening length defined within a Yukawa potential, and $W$ the Debye-Waller factor).

For mono-layer graphene, the lattice sum simplifies to

$$
S_{b}\left(v_{1}, v_{2}\right)=2 \cos \left[\frac{\pi}{3}\left(v_{1}+v_{2}\right)\right],
$$

where $v_{1}$ and $v_{2}$ are the integer indices of specific reflections in terms of the graphene reciprocal lattice vectors. The square of the lattice sum (2) then takes a value of either 1 or
4 depending on $v_{1}$ and $v_{2}$ and hence defines "weak" and "strong" reflections, respectively.

The DWF is included in (1) to account for the attenuation of scattering due to thermal atomic motion. For monolayer graphene, this can be expressed as

$$
2 W=\Delta \boldsymbol{k}_{p}^{2} u_{p}^{2}+\Delta \boldsymbol{k}_{\perp}^{2} u_{\perp}^{2},
$$

where $u_{p}$ and $u_{\perp}$ are the mean displacement of atoms from their ideal lattice positions in directions parallel $\left(u_{p}\right)$ and perpendicular $\left(u_{\perp}\right)$ to the incident electron beam. For a diffraction pattern from mono-layer graphene recorded at normal incidence, only atomic displacements parallel to the basal plane will directly contribute to the measured DWF and hence $u_{\perp}^{2}$ can be effectively ignored. ${ }^{14}$ Out-of-plane vibrations will however affect the projected atomic positions in the mono-layer graphene lattice and consequently contribute to an effective in-plane component in the measured DWF.

We first validate the use of (1) to determine the in-plane mean square atomic displacement, by verifying that kinematical scattering theory is satisfied for mono-layer graphene. Using a multi-slice calculation ${ }^{23}$ (see supplementary information $^{24}$ ), electron diffraction patterns from mono-layer graphene were simulated for both kinematic and fully dynamical electron scattering. In the kinematic case, the whole atomic potential is projected onto a single simulation slice, whereas for the dynamical case, the atomic potential is sub-sampled in slices of a fraction of its diameter. ${ }^{25}$ No difference was found for these two extreme cases (see supplementary Figure $\mathrm{S}^{24}$ ), supporting the assumption that at the voltage used kinematic scattering is valid for mono-layer graphene.

To analyze the experimental diffraction pattern, we have followed the methodology set out in Ref. 14. Each reflection in the diffraction pattern was first located using a semiautomated peak finding procedure in which the locations of the first order reflections were initially manually estimated and their positions subsequently refined by fitting a two dimensional Gaussian to each peak. The reciprocal lattice vectors are defined in this initial step (Figure 1(a)) and can be subsequently used to automatically locate all other reflections in the diffraction pattern. A two dimensional Gaussian (a)

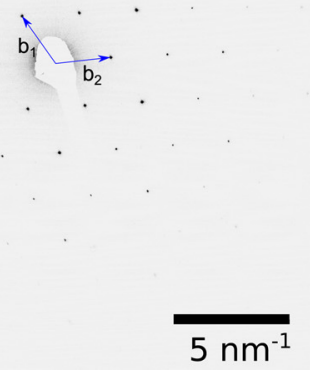

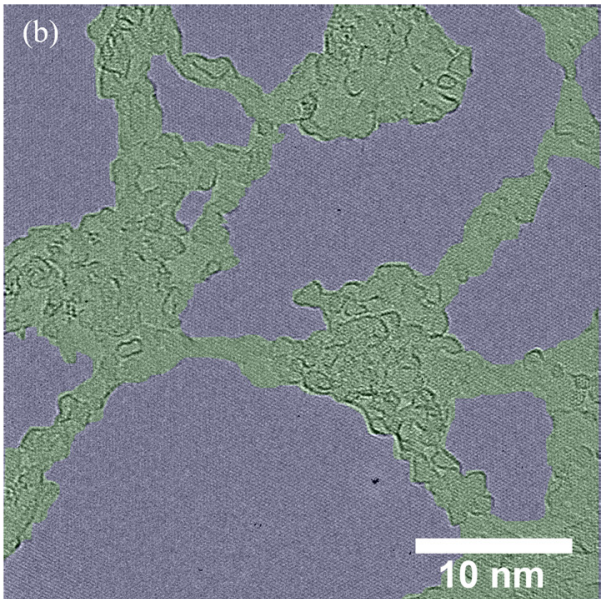

FIG. 1. (a) Selected area diffraction pattern of mono-layer graphene recorded at $310 \mathrm{~K}$ and zero tilt angle. Reciprocal lattice vectors $b_{1}$ and $b_{2}$ are marked. (b) False colour HRTEM image of the graphene sample. Approximate areas of pristine graphene are coloured blue and approximate areas of amorphous contaminants coloured green. 
(a)

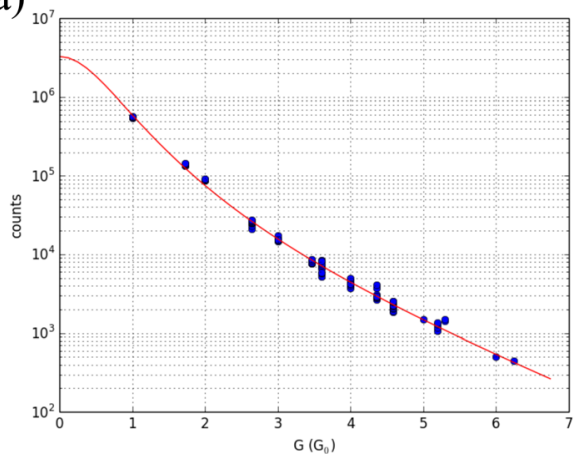

(b)

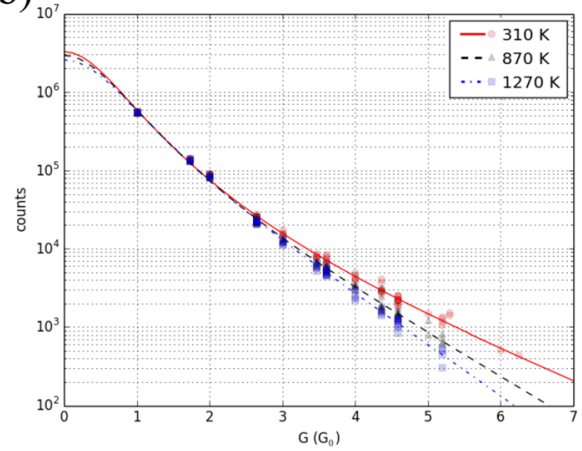

FIG. 2. (a) Total recorded CCD counts as a function of scattering angle for all recorded peaks in the diffraction pattern shown in Figure 1(a). The counts for "strong" peaks (defined by (2)) have been divided by four. The red line is a least square fit to (1). (b) Comparison of least squares fits to the data taken at $\sim 310 \mathrm{~K}, 870 \mathrm{~K}$, and $1270 \mathrm{~K}$. was then fitted to each peak position, a planar background subtracted and counts summed over an area around the peak of eight times the full width half-maximum of the Gaussian fit. Using this procedure, measuring total counts rather than peak height, the analysis reported remains robust to extraneous experimental factors that may cause peak broadening, such as rippling of the mono-layer graphene sheet or small sample tilts away from the normal to the incident beam. ${ }^{10}$ The number of counts recorded in "strong" peaks (defined by (2)) was then divided by four, thus removing the lattice sum from the analysis.

The total number of counts in each reflection as a function of scattering angle $(G)$ for mono-layer graphene at normal incidence is shown in Figure 2(a). The red line shows a least squares fit to (1) with three free parameters corresponding to the incident beam current, DWF, and inverse Coulomb screening length. For mono-layer graphene at $T \sim$ $310 \mathrm{~K}$ and zero tilt angle, we calculate values for the inplane mean square atomic displacement, $u_{p}^{2}=15 \pm 1 \mathrm{pm}^{2}$ and an inverse coulomb screening length, $\mu^{-1}=39 \pm 2 \mathrm{pm}$ with uncertainties in both measurements calculated from the co-variance matrix returned from the least squares fitting procedure. $^{26}$

This measured value of $u_{p}^{2}$ is due to both dynamic disorder due to phonons and static disorder due to defects in the crystal lattice. We have extensively studied the graphene used in this experiment by means of high resolution transmission electron microscopy and have found it to have a very low intrinsic defect density. ${ }^{27}$ We therefore attribute the measured value of $u_{p}^{2}$ to dynamic disorder due to phonons.

As previously noted, out-of-plane vibrations in the mono-layer graphene lattice can alter the projected in-plane atomic positions and thus potentially contribute to the measured value of $u_{p}^{2}$. Out-of-plane corrugations in mono-layer graphene have been independently estimated to have amplitudes $\sim 1 \mathrm{~nm}$ and length scales of $\sim 25 \mathrm{~nm} .{ }^{10}$ Using these values and assuming a fixed carbon-carbon bond length and a simple linear atomic chain model, we estimate that the contribution from out-of-plane corrugations is of the order of $0.5 \mathrm{pm}^{2}$, approximately half the experimental uncertainty in our measured value.

The value obtained from our data for the inverse Coulomb screening length, $\mu^{-1}=39 \pm 2 \mathrm{pm}$, is larger than that which has been previously experimentally measured $(34 \pm 2 \mathrm{pm}){ }^{14}$ The Coulomb screening length is dependent on the electron density in the material ${ }^{28}$ and will therefore be affected by the level of doping in the graphene sample. We believe this may account for the discrepancy between our results and those previously published.

Figure 1(b) shows the best fit to diffraction patterns taken at $\sim 310 \mathrm{~K}, 810 \mathrm{~K}$, and $1270 \mathrm{~K}$. As expected, an increase in temperature results in a faster decay of the measured counts with scattering angle. We attribute this to an increase in the in-plane thermal motion of the carbon atoms with a concomitant increase in diffuse scattering. Under the broad-beam conditions used to collect these diffraction patterns, we believe that any heating due to the incident electron beam is only a small contribution to the sample temperature.

Thirteen diffraction patterns were also recorded at temperatures from $\sim 100 \mathrm{~K}$ to $\sim 1270 \mathrm{~K}\left(-170{ }^{\circ} \mathrm{C}\right.$ to $\left.1000^{\circ} \mathrm{C}\right)$ and in-plane mean-square displacement extracted as described previously. Figure 3 shows the variation of $u_{p}^{2}$ with temperature (see supplementary information ${ }^{24}$ for tabulated values). At $\mathrm{T} \lesssim 400 \mathrm{~K}$, the value of $u_{p}^{2}$ is consistent (within experimental uncertainties) with previously reported calculations of the in plane quantum mechanical zero point vibrations for mono-layer graphene of $16 \mathrm{pm}^{2} .{ }^{13}$ This indicates that at temperature up to $\sim 400 \mathrm{~K}$, the zero-point energy contribution dominates the population of phonon states in mono-layer graphene and that the zero-point energy vibrations in mono-layer graphene are sufficiently large to account for experimentally

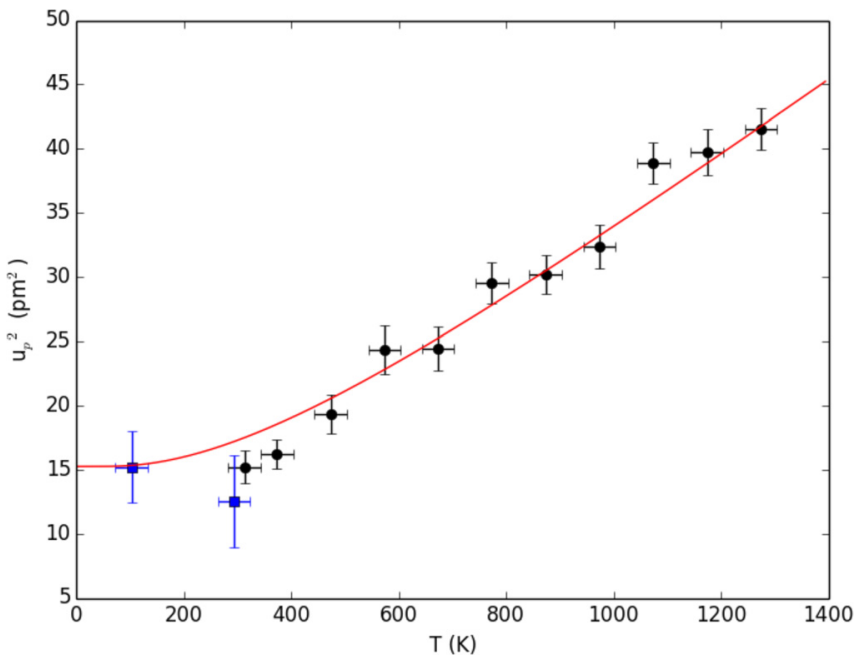

FIG. 3. Measured in-plane mean square displacement $u_{p}^{2}$ for temperatures from $\sim 100 \mathrm{~K}$ to $\sim 1270 \mathrm{~K}$. Blue data points (square markers) were measured with a liquid nitrogen holder on a different mono-layer graphene sample. The red line is a fit to (5). 
reported room-temperature phonon-mediated displacement cross-sections. ${ }^{29}$

It has been shown that when heated to $>600 \mathrm{~K}$, defects in graphene are repaired due to mobile carbon atoms on the crystal surface. ${ }^{30,31}$ If defects are a major contribution to our determined value of $u_{p}^{2}$, we would expect a kink in the measured temperature dependence at the point at which the defects are annealed out of the graphene crystal. The smooth increase in $u_{p}^{2}$ with temperature supports our earlier assertion that we are primarily measuring dynamic disorder due to phonons.

The red line in Figure 3 is a fit to the analytical expression

$$
2 W=2 \frac{G^{2}}{k_{D}^{2}} \frac{k_{B} T}{M v_{s}^{2}}\left[\frac{x_{D}-x_{s}}{2}+\ln \left(\frac{1-e^{-x_{D}}}{1-e^{-x_{S}}}\right)\right],
$$

which has been derived by considering a simple Debye model for the phonon density of states in mono-layer graphene ${ }^{14}$ and where $k_{D}$ is the Debye wave vector $\left(k_{D}=\frac{\Theta_{D} k_{B}}{\hbar v_{s}}\right.$ with $\Theta_{D} \sim$ $2300 \mathrm{~K}$ the Debye temperature for planar phonons in monolayer graphene $\left.\mathrm{e}^{13}\right), M$ is the mass of a carbon atom, $v_{s}=2.2 \times 10^{4} \mathrm{~ms}^{-2}$, the speed of sound in mono-layer graphene, and $k_{B} T$ the thermal energy. In order to avoid a divergence of the DWF at any non-zero temperature, a minimum phonon wave-vector that can be supported by the crystal, $k_{s}$ is introduced, ${ }^{13}$ with $x_{s}=\frac{\hbar v_{s} k_{s}}{k_{B} T}$ the ratio of the energy associated with $k_{s}$ to the thermal energy. Similarly, $x_{D}=\frac{\hbar v_{S} k_{D}}{k_{B} T}$ is the ratio of the Debye to thermal energies.

Expanding (4) gives

$$
2 W=G^{2}\left[\frac{\hbar}{k_{D} M v_{s}}\left(1-\frac{k_{S}}{k_{D}}\right)+\frac{2 k_{B} T}{k_{D}^{2} M v_{s}^{2}} \ln \left(\frac{1-e^{-x_{D}}}{1-e^{-x_{S}}}\right)\right] .
$$

The first term in (5) corresponds to a temperature independent zero point motion. Within the model described, the magnitude of the zero-point contribution to the mean-square atomic displacement depends on the ratio $k_{S} / k_{D}$. For values of $k_{S}=k_{D}$, the zero-point contribution vanishes and for $k_{S}>k_{D}$, it becomes negative. This is clearly unphysical and therefore sets a limit on the validity of (5) which is only physically meaningful for $k_{s}<k_{D}\left(\frac{k_{s}}{k_{D}}<1\right)$. If we further assume that the minimum phonon wave-vector (maximum wavelength) $k_{s}=\frac{2 \pi}{\lambda_{s}}$ is limited by the effective crystal size $L$ (with $\lambda_{s}=L$ ), then for planar phonons in mono-layer graphene this Debye model should hold for crystal dimensions of $L>5 \times 10^{-10} \mathrm{~m}$.

A least squares fit of (5) to the experimentally determined values for $u_{p}^{2}$ gives $k_{s}=1.8 \pm 0.2 \times 10^{9} \mathrm{~m}^{-1}$ corresponding to a maximum phonon wavelength (effective crystal size), $\lambda_{s}=3.5 \pm 0.4 \times 10^{-9} \mathrm{~m}$. We have previously measured the single crystal grain size in the CVD monolayer graphene used for the measurements reported to have dimensions of the order of hundreds of microns ${ }^{17}$ with very low intrinsic defect densities. ${ }^{27} \mathrm{~A}$ similar analysis of SAED patterns from exfoliated graphene yields a value of $u_{p}^{2}=40 \pm 10 \mathrm{pm}^{2}$ at $300 \mathrm{~K}$ and a corresponding maximum phonon wavelength of similar dimensions to the exfoliated crystallite size $(\sim 3.5 \mu \mathrm{m}) .{ }^{14}$ However for CVD mono-layer graphene, we find a large disparity between the determined "effective" and the measured "real" crystal size. It is important to note that the maximum phonon wavelength of $\lambda_{s}=3.5 \pm 0.2 \times 10^{-9} \mathrm{~m}$ determined is at the limit of the range within which (5) is physically meaningful.

We have also measured the temperature dependence of $u_{p}^{2}$ for three different samples (Figure $\mathrm{S}^{24}$ ). Each sample shows broadly similar values of $u_{p}^{2}$ across the temperature range used for the measurements, indicating that the low determined effective crystal size is not due to the specific local environment of the sample but is indicative of the CVD grown mono-layer graphene.

It is conceptually possible that the low measured effective crystal size is due to damping of long-wavelength phonons by adsorbates on the surface of the sample. Real space imaging of the sample used shows that at room temperature, the lateral dimensions of adsorbate free regions are of the order of 3-50 nm (Figure 1(b)). However at elevated temperatures $(\mathrm{T} \gtrsim 800 \mathrm{~K})$, the mono-layer graphene is increasingly free of surface contamination (Figure S2). Hence, if damping due to surface adsorbates was significantly affecting our measured values of $u_{p}^{2}$, we would expect to observe a rapid increase in $u_{p}^{2}$ at high temperatures as the mono-layer graphene becomes increasingly free of surface adsorbates. However, the measured temperature dependence of $u_{p}^{2}$ remains approximately linear at high temperatures and we therefore conclude that damping due to surface adsorbates is not the cause of the low measured value of $\lambda_{s}$.

A phonon with wavelength significantly larger than the area illuminated by the electron beam will not introduce effective disorder but instead will manifest as a displacement (a)

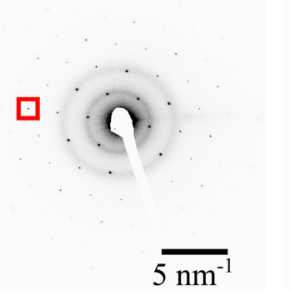

(b)

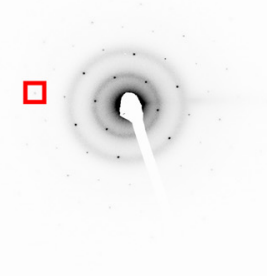

(c)

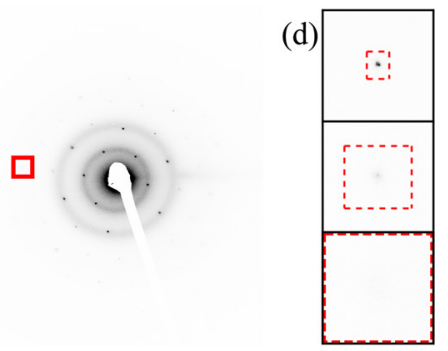

FIG. 4. Selected area diffraction patterns from mono-layer graphene recorded at $T \sim 310 \mathrm{~K}$ and with sample tilts of (a) $0^{\circ}$, (b) $-6^{\circ}$, and (c) $-14^{\circ}$. The red boxes highlight the area around the diffraction peaks (in this case the $(3, \overline{3}, 0,0)$ peak) used for background subtraction. (d) Magnified images of the $(3, \overline{3}, 0,0)$ diffraction peak at tilt angles of $0^{\circ}$ (top), $-6^{\circ}$ (middle), and $-14^{\circ}$ (bottom). The dashed boxes correspond to the area around each peak within which the total number of counts are determined. 
of the entire lattice. Even within the extent of the area illuminated by the electron beam disorder will only be evident in the diffraction pattern at distances less than the spatial coherence of the beam. For coherent plane wave illumination, the intensity of the reflections is insensitive to lattice displacements $^{32}$ and therefore the mean square displacements calculated in this work can be due only to phonons with wavelengths of the order of, or shorter than, the partial spatial coherence length of the illuminating beam. The value of $\lambda_{s}=3.5 \pm 0.2 \times 10^{-9} \mathrm{~m}$ is of the same order of magnitude as the partial spatial coherence width (at the specimen plane) previously measured for this microscope ${ }^{33}$ (albeit in a different optical configuration).

The Debye approximation of a linear relationship between wave-vector and phonon energy used in the calculation of (4) and (5) is reasonable for longitudinal acoustic (LA) and transverse acoustic (TA) phonons in mono-layer graphene at $T<900 \mathrm{~K} .{ }^{34}$ Above this temperature, the dispersion relations for LA and TA phonons deviate significantly from linearity. However, even at $1500 \mathrm{~K}$, the population of phonons within the non-linear region $\left(k>10^{8} \mathrm{~m}^{-1}\right)$ is only a few percent of the population of phonons in the linear region $\left(k<10^{8} \mathrm{~m}^{-1}\right)$. This small contribution of phonons within the non-linear region of the dispersion relation, even at temperatures well above those achieved in our experiments justifies the use of the Debye approximation in the calculation of $u_{p}^{2}$ across the temperature range explored.

Due to the kinematical nature of diffraction from monolayer graphene, diffraction patterns recorded at normal incidence are insensitive to out-of-plane vibrations of the crystal lattice. For mono-layer graphene, these flexural modes dominate thermal transport ${ }^{35}$ and also have a large impact on electronic properties. If the basal plane is tilted away from normal to the incident electron beam, both the in-plane and out-of-plane vibrations will contribute to the measured DWF. Under this condition the total mean square displacement normal to the incident electron beam is simply equal to

$$
u_{p}^{2}=\left(u_{x y} \cos \theta\right)^{2}+\left(u_{z} \sin \theta\right)^{2}
$$

where $u_{x y}$ and $u_{z}$ are the planar and flexural mean atomic displacement, respectively, and $\theta$ the tilt angle.

Figures 4(a)-4(c) show diffraction patterns recorded at $T \sim 310 \mathrm{~K}$ with the sample titled away from normal to the incident beam by between $0^{\circ}$ and $14^{\circ}$. As the graphene basal plane is tilted, the intensity and number of recorded counts of the diffraction peaks decrease (see Figure 4(d)) with this effect being more pronounced for higher order peaks. This is in part caused by an increase in DWF due to contributions from flexural phonons.

Figures 5(a)-5(c) show the measured DWF for monolayer graphene as a function of tilt angle at $\mathrm{T} \sim 310 \mathrm{~K}$, $770 \mathrm{~K}$, and $1270 \mathrm{~K}$, respectively. Due to fewer measurable peaks at high tilt angle and the sensitivity of this analysis to the high order peaks, the value for the inverse Coulomb screening length was fixed using the value determined from fits to the zero tilt data. Fitting (5) to the measured DWF as a function of tilt angle enables both the planar and flexural mean square atomic displacements to be determined (Table I).

At all temperatures, the values determined for $u_{z}^{2}$ are over an order of magnitude greater than $u_{x y}^{2}$. Theoretical calculations of the zero temperature in-plane and flexural mean

(a)

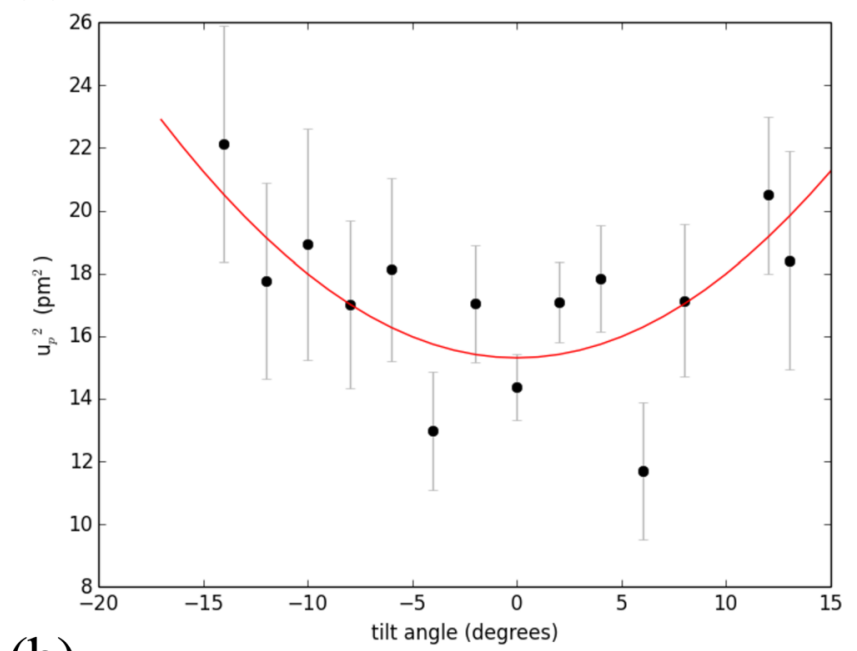

(b)

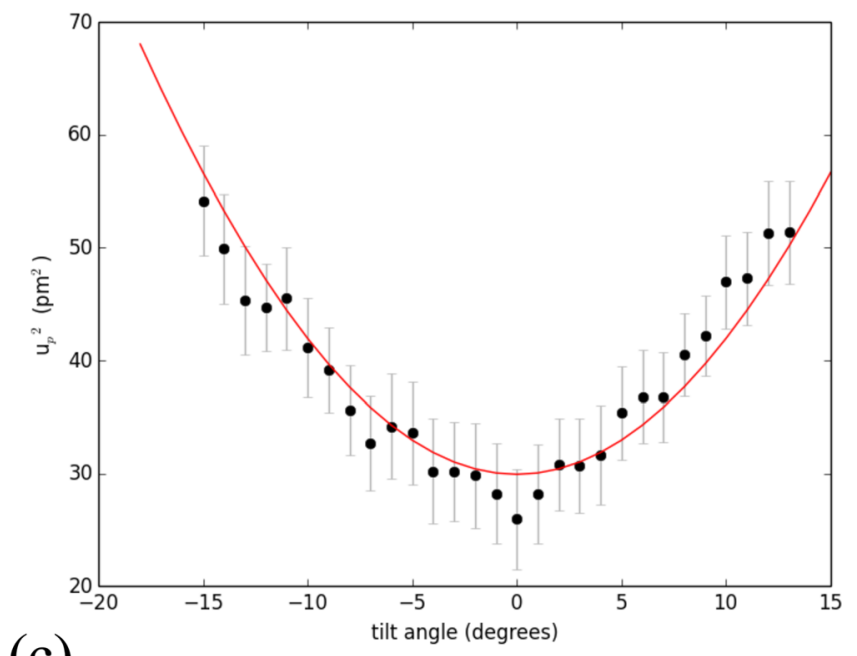

(c)

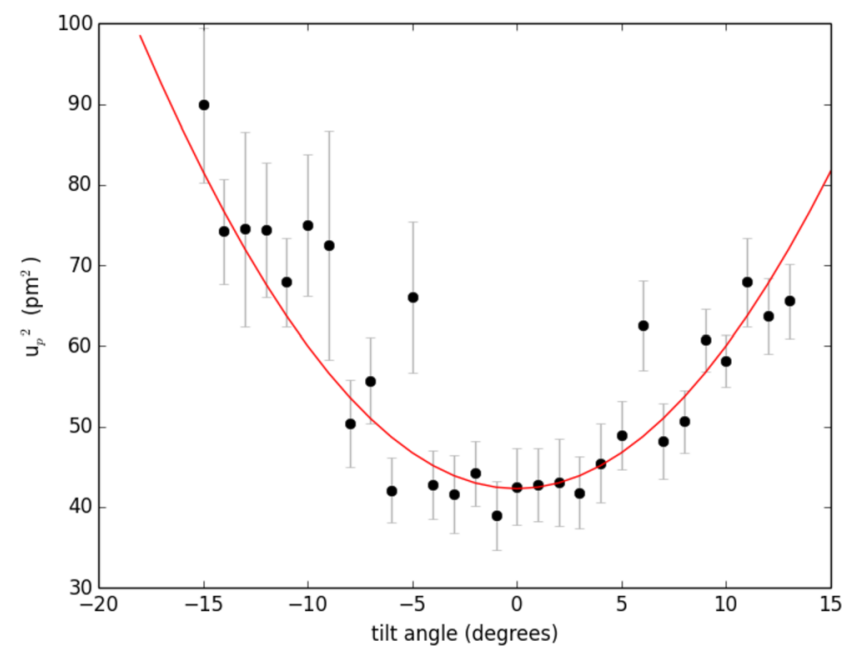

FIG. 5. Debye-Waller factor as a function of specimen tilt angle measured at (a) $310 \mathrm{~K}$, (b) $770 \mathrm{~K}$, and (c) $1270 \mathrm{~K}$. The red lines are fits to (6). 
TABLE I. Temperature dependence of planar and flexural mean square atomic displacements.

\begin{tabular}{lcc}
\hline \hline Temperature $(\mathrm{K})$ & $u_{x y}^{2}\left(\mathrm{pm}^{2}\right)$ & $u_{z}^{2}\left(\mathrm{pm}^{2}\right)$ \\
\hline 310 & $15.2 \pm 0.8$ & $104 \pm 40$ \\
770 & $30.3 \pm 0.6$ & $430 \pm 20$ \\
1270 & $39.7 \pm 0.6$ & $630 \pm 30$ \\
\hline \hline
\end{tabular}

square atomic displacements have predicted values of $u_{x y}^{2}(0 K)=15.9 \mathrm{pm}^{2}$ and $u_{z}^{2}(0 K)=40.4 \mathrm{pm}^{2}{ }^{13}$ While our value for $u_{x y}^{2}(310 \mathrm{~K})$ is consistent with that predicted due to zero-point motion alone, $u_{z}^{2}(310 \mathrm{~K})$ is approximately 2.5 times the predicted zero-point energy value. This indicates that in contrast to the in-plane case, at $310 \mathrm{~K}$, there is a significant thermal contribution to the population of out-ofplane (flexural) phonon states.

Figure 6 shows the relative increase in $u_{x y}^{2}$ and $u_{z}^{2}$ with temperature normalized to the $\mathrm{T}=310 \mathrm{~K}$ values. For a temperature increase of almost $1000 \mathrm{~K}$, the values for $u_{x y}^{2}$ increase by approximately a factor of two, while $u_{z}^{2}$ increases by more than a factor of six. We attribute this faster rise of $u_{z}^{2}$ with temperature to the approximately quadratic dispersion relation of the flexural phonons in mono-layer graphene in the temperature range studied. ${ }^{36}$

The dispersion relation for flexural phonons in monolayer graphene can be approximated as $\omega(k) \sim \alpha k^{2}$ with $\alpha=6.2 \times 10^{-7} \mathrm{~m}^{2} / \mathrm{s}^{35}$ Using this approximation, we can derive an expression for the out-of-plane or flexural mean square atomic displacement ${ }^{24}$ as

$$
u_{z}^{2}=\frac{\hbar}{2 M k_{D}^{2} \alpha} \int_{x_{s}}^{x_{D}} \frac{1}{x}\left[\frac{1}{\exp (x)-1}+\frac{1}{2}\right] d x,
$$

for which $x=\hbar \alpha k^{2} / k_{B} T$ and the integral is bounded by the smallest wave-vector supported by the crystal $\left(k=k_{S}\right)$ and the Debye wave-vector $\left(k=k_{D}\right)$, which for flexural phonons in mono-layer graphene is equal to $k_{D}=7.6 \times 10^{9}$ (corresponding to a Debye temperature of $\left.\Theta_{D}=1287 \mathrm{~K}\right) .{ }^{13}$

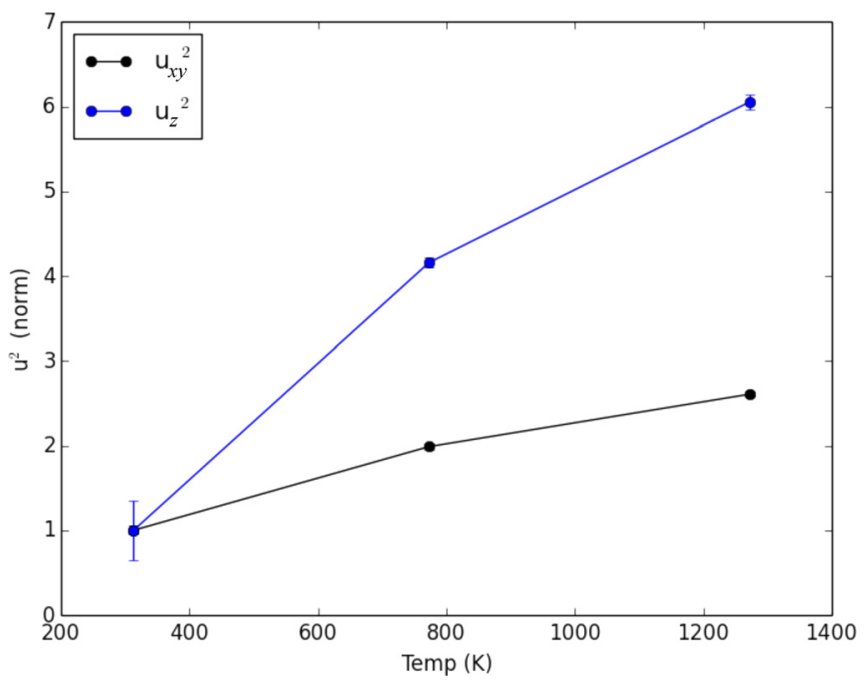

FIG. 6. Temperature dependence of the planar $\left(u_{x y}^{2}\right)$ and flexural $\left(u_{z}^{2}\right)$ mean square displacements normalized to their $\mathrm{T}=310 \mathrm{~K}$ values.

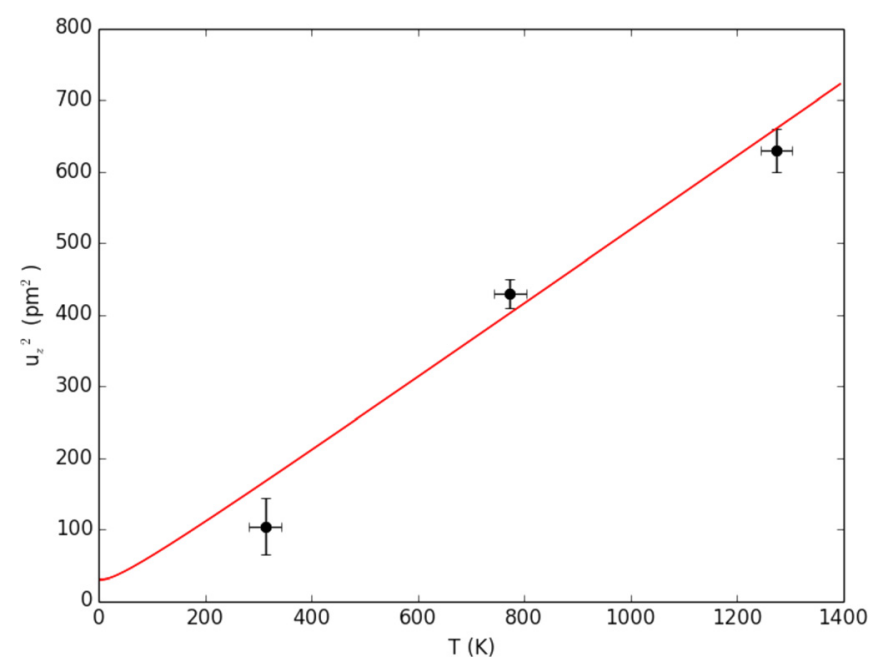

FIG. 7. Temperature dependence of the flexural mean square atomic displacement $u_{z}^{2}$. The red line is a fit to Eq. (7).

The temperature dependence of the flexural mean square atomic displacement, $u_{z}^{2}$, is shown in Figure 7. A fit to (7) gives a value for the longest wavelength flexural phonon sampled of $\lambda_{s, z}=2.5 \pm 0.3 \times 10^{-9} \mathrm{~m}$, somewhat smaller than the value determined from the in-plane contribution to the DWF $\left(\frac{\lambda_{s, z}}{\lambda_{s, x y}} \approx 0.7\right)$. Again, this value for $\lambda_{s, z}$ is remarkably small considering the physical dimensions of the mono-layer graphene crystallite used for the measurement.

We have also calculated a value for the quantum mechanical zero point motion of the flexural phonons of $u_{z}^{2}(0 K)=30 \pm 1 \mathrm{pm}^{2}$ which is 0.75 times the theoretically predicted value of $u_{z}^{2}(0 K)=40.4 \mathrm{pm}^{2}$. $^{13}$

It is important to note that due to the sparsity of data points used in the calculation of $\lambda_{s, z}$ and $u_{z}^{2}(0 K)$ (see Figure 7), the experimental uncertainty in these physical parameters has been significantly underestimated. Further work is required to accurately determine these parameters.

\section{CONCLUSION}

We have experimentally measured the mean square inplane and out-of-plane atomic displacements for mono-layer graphene across a wide temperature range. By fitting the temperature dependence of $u_{x y}^{2}$ to a Debye-model, we have calculated a value for the longest wavelength planar phonon of $\lambda_{s, x y}=3.5 \pm 0.4 \times 10^{-9} \mathrm{~m}$. This is interpreted as the upper limit to the phonon wavelength being sampled in the experiment and is most likely set by the partial spatial coherence of the incident electron beam. At temperatures less than $400 \mathrm{~K}$, our measured value for $u_{x y}^{2}=15 \pm 1 \mathrm{pm}^{2}$ is consistent with a theoretically calculated value of $u_{x y}^{2}=16 \mathrm{pm}^{2}$ due only to quantum mechanical zero-point motion.

The values for $u_{z}^{2}$ measured from tilt series diffraction experiments are over an order of magnitude larger than $u_{x y}^{2}$, confirming the softer nature of flexural phonons in monolayer graphene. Moreover, the experimentally determined value of $u_{z}^{2}(310 \mathrm{~K})=104 \pm 40 \mathrm{pm}^{2}$ is over twice that theoretically predicted for zero-point motion alone $\left(u_{z}^{2}=40 \mathrm{pm}^{2}\right)$. Assuming a quadratic dispersion relation for flexural phonons in mono-layer graphene, we have developed an expression for 
the magnitude of the out-of-plane mean square displacement, $u_{z}^{2}$. Fitting the experimental data to this expression, we have determined a value for the longest wavelength flexural phonon sampled of $\lambda_{s, z}=2.5 \pm 0.3 \times 10^{-9} \mathrm{~m}$, and a value for the mean square amplitude of quantum mechanical zero point motion of $u_{z}^{2}(0 K)=30 \pm 1 \mathrm{pm}^{2}$.

The experimentally determined values of the DebyeWaller factor in mono-layer graphene reported in this work will help in the quantitative understanding of a wide range of scattering and imaging experiments, including the interpretation of high resolution TEM images. Furthermore, these results may provide insight into the mechanisms responsible for stabilizing two dimensional crystals.

\section{ACKNOWLEDGMENTS}

Financial support from EPSRC (Platform Grant Nos. EP/F048009/1 and EP/K032518/1) and the EU ESTEEM2 (Enabling Science and Technology through European Electron Microscopy) project (7th Framework Programme of the European Commission) is acknowledged.

${ }^{1}$ R. E. Peierls, Helv. Phys. Acta 7, 81 (1923).

${ }^{2}$ L. D. Landau, Phys. Z. Sowjetunion 11, 26 (1937).

${ }^{3}$ N. Mermin, Phys. Rev. 176, 250 (1968).

${ }^{4}$ K. S. Novoselov, A. K. Geim, S. V. Morozov, D. Jiang, Y. Zhang, S. V. Dubonos, I. V. Grigorieva, and A. A. Firsov, Science 306, 666 (2004).

${ }^{5}$ K. S. Novoselov, D. Jiang, F. Schedin, T. J. Booth, V. V. Khotkevich, S. V. Morozov, and A. K. Geim, Proc. Natl. Acad. Sci. U.S.A. 102, 10451 (2005).

${ }^{6}$ A. Kara, H. Enriquez, A. P. Seitsonen, L. C. Lew Yan Voon, S. Vizzini, B. Aufray, and H. Oughaddou, Surf. Sci. Rep. 67, 1 (2012).

${ }^{7}$ Q. Tang and Z. Zhou, Prog. Mater. Sci. 58, 1244 (2013).

${ }^{8}$ A. O'Hare, F. V. Kusmartsev, and K. I. Kugel, Nano Lett. 12, 1045 (2012).

${ }^{9}$ A. Fasolino, J. H. Los, and M. I. Katsnelson, Nature Mater. 6, 858 (2007).

${ }^{10}$ J. C. Meyer, A. K. Geim, M. I. Katsnelson, K. S. Novoselov, T. J. Booth, and S. Roth, Nature 446, 60 (2007).

${ }^{11}$ L. Reimer and H. Kohl, Transmission Electron Microscopy, 5th ed. (Springer, 2008).
${ }^{12}$ A. A. Maradudin, E. W. Montroll, G. H. Weiss, and I. P. Ipatova, Theory of Lattice Dynamics in the Harmonic Approximation, 2nd ed. (Academic, New York, 1971).

${ }^{13}$ V. Tewary and B. Yang, Phys. Rev. B 79, 125416 (2009).

${ }^{14}$ B. Shevitski, M. Mecklenburg, W. A. Hubbard, E. R. White, B. Dawson, M. S. Lodge, M. Ishigami, and B. C. Regan, Phys. Rev. B 87, 045417 (2013).

${ }^{15}$ M. J. Hÿtch and W. M. Stobbs, Ultramicroscopy 53, 191 (1994).

${ }^{16}$ A. Howie, Ultramicroscopy 98, 73 (2004).

${ }^{17}$ Y. A. Wu, Y. Fan, S. Speller, G. L. Creeth, J. T. Sadowski, K. He, A. W. Robertson, C. S. Allen, and J. H. Warner, ACS Nano 6, 5010 (2012).

${ }^{18}$ J. F. Creemer, D. Briand, H. W. Zandbergen, W. van der Vlist, C. R. de Boer, N. F. de Rooij, and P. M. Sarro, Sens. Actuators, A 148, 416 (2008).

${ }^{19}$ M. Mukai, J. S. Kim, K. Omoto, H. Sawada, A. Kimura, A. Ikeda, J. Zhou, T. Kaneyama, N. P. Young, J. H. Warner, P. D. Nellist, and A. I. Kirkland, Ultramicroscopy 140, 37 (2014).

${ }^{20}$ N. P. Young, M. A. van Huis, H. W. Zandbergen, H. Xu, and A. I. Kirkland, Ultramicroscopy 110, 506 (2010).

${ }^{21}$ J. C. Meyer, A. K. Geim, M. I. Katsnelson, K. S. Novoselov, D. Obergfell, S. Roth, C. Girit, and A. Zettl, Solid State Commun. 143, 101 (2007).

${ }^{22}$ A. Rose, Adv. Electron. 1, 131 (1948).

${ }^{23}$ J. M. Cowley and A. F. Moodie, Acta Crystallogr. 10, 609 (1957).

${ }^{24}$ See supplemental material at http://dx.doi.org/10.1063/1.4928324 for details of electron diffraction pattern simulations, tabulated values of $u_{p}^{2}$, results from additional samples, high resolution TEM images of heated monolayer graphene, and derivation of $u_{z}^{2}$.

${ }^{25}$ I. Lobato and D. Van Dyck, Ultramicroscopy 156, 9 (2015).

${ }^{26}$ P. H. Richter, TDA Prog. Rep. 42-122, 107-137 (1995).

${ }^{27}$ A. W. Robertson, C. S. Allen, Y. A. Wu, K. He, J. Olivier, J. Neethling, A. I. Kirkland, and J. H. Warner, Nat. Commun. 3, 1144 (2012).

${ }^{28}$ N. W. Ashcroft and N. D. Mermin, Solid State Physics (Brooks/Cole, Cenage Learning, 1976).

${ }^{29}$ J. C. Meyer, F. Eder, S. Kurasch, V. Skakalova, J. Kotakoski, H. J. Park, S. Roth, A. Chuvilin, S. Eyhusen, G. Benner, A. V. Krasheninnikov, and U. Kaiser, Phys. Rev. Lett. 108, 196102 (2012).

${ }^{30}$ J. Chen, T. Shi, T. Cai, T. Xu, L. Sun, X. Wu, and D. Yu, Appl. Phys. Lett. 102, 103107 (2013).

${ }^{31}$ L. Sun, F. Banhart, and J. Warner, MRS Bull. 40, 29 (2015).

${ }^{32}$ A. V. Martin, L. J. Allen, and K. Ishizuka, Ultramicroscopy 110, 359 (2010).

${ }^{33}$ C. Dwyer, A. I. Kirkland, P. Hartel, H. Müller, and M. Haider, Appl. Phys. Lett. 90, 151104 (2007).

${ }^{34}$ D. Nika, E. Pokatilov, A. Askerov, and A. Balandin, Phys. Rev. B 79, 155413 (2009).

${ }^{35}$ L. Lindsay, D. A. Broido, and N. Mingo, Phys. Rev. B 82, 115427 (2010).

${ }^{36}$ E. Pop, V. Varshney, and A. K. Roy, MRS Bull. 37, 1273 (2012). 